\title{
HOPF FIBRATIONS ON LENS SPACES
}

\author{
SUNGBOK HONG
}

ABSTRACT. We give a certain uniqueness properties for the fiber of the Hopf fibration on lens spaces.

\section{Introduction}

Let $S^{3}$ be the unit sphere in $\mathbb{C} \times \mathbb{C}$ endowed with the geometry associated with the natural action of $O(4)$. Let $p, q$ be relatively prime positive integers. The map $\rho: S^{3} \rightarrow S^{3}$ defined by $\rho(u, v)=\left(e^{2 \pi i q / p} u, e^{-2 \pi i / p} v\right)$ is an isometry which generates a free $\mathbb{Z}_{p}$ action on $S^{3}$. The quotient space $S^{3} /\langle\rho\rangle$ is the lens space $L(p, q)$. We let $\mu: S^{3} \rightarrow L(p, q)$ denote the quotient map. The 3 sphere $S^{3}$ is the union of two solid tori $V_{1}=\left\{(u, v) \in S^{3}:|u|^{2} \geq 1 / 2\right\}$ and $V_{2}=\left\{(u, v) \in S^{3}:|u|^{2} \leq 1 / 2\right\}$ whose intersection is the torus $T=\left\{(u, v) \in S^{3}:|u|^{2}=1 / 2\right\}$. This decomposition of $S^{3}$ is invariant under $\rho$ and descends to give a decomposition of $L(p, q)$ into solid tori $\mu\left(V_{1}\right), \mu\left(V_{2}\right)$ whose intersection is the torus $\mu(T)$. Choose integers $r$ and $s$ so that $r q-p s=-1$, and $f$ be the affine diffeomorphism on $S^{1} \times S^{1}$ given by $f(u, v)=\left(u^{r} v^{p}, u^{s} v^{q}\right)$. Then $L(p, q)$ can also be described as the 3-manifold $V \cup_{f} V$ obtained by identifying the boundaries of a solid torus $V=S^{1} \times D^{2}$ using $f: \partial V \rightarrow \partial V$ as attaching map. For more details on these definitions see [2], [3] and [5].

An embedded torus which separates $L(p, q)$ into two solid tori $V_{1}$ and $V_{2}$ is called a Heegaard torus, and the associated decomposition of the lens space into two solid tori is a Heegaard decomposition. Bonahon [1] has shown that any two Heegaard tori of a lens space are isotopic. Hence every diffeomorphism of $L(p, q)$ is isotopic to a diffeomorphism which preserves the Heegaard torus and Bonahon used this idea to calculate the mapping class group of $L(p, q)$, namely $\pi_{0} \operatorname{Diff}(L(p, q))$.

Received June 23, 2003.

2000 Mathematics Subject Classification: $57 \mathrm{M} 99$.

Key words and phrases: lens space, Hopf fibration, Heegaard torus.

Supported by Korea University. 
A sweepout of $L(p, q)$ is a smooth map $\sigma: T \times[0,1] \rightarrow L(p, q)$, where $T$ is a torus such that

1. $\Sigma_{0}=\sigma(T \times\{0\})$ and $\Sigma_{1}=\sigma(T \times\{1\})$ are imbedded circles in $L(p, q)$,

2. $\left.\sigma\right|_{T \times(0,1)}: T \times(0,1) \rightarrow L(p, q)$ is a diffeomorphism onto $L(p, q)-$ $\left(\Sigma_{0} \cup \Sigma_{1}\right)$

3. Near $T \times \partial I, \sigma$ gives a mapping cylinder neighborhood of $\Sigma_{0} \cup \Sigma_{1}$.

Associated to any $t$ with $0<t<1$, we denote $\sigma(T \times t)$ by $P_{t}$, and call it a level surface of $\sigma$.

The standard elliptic geometry on the 3-sphere is the geometry associated with the orthogonal group under its natural action on the unit sphere in $\mathbb{R}^{4}$. A 3-manifold $M$ is elliptic if it admits a covering map $S^{3} \rightarrow M$ whose covering transformations act freely on $S^{3}$ as a subgroup of $\operatorname{Isom}\left(S^{3}\right)=O(4)$. The Hopf fibering on $S^{3}$ is an $S^{1}$-bundle structure with projection map $H: S^{3} \rightarrow S^{2}=\mathbb{C} \cup\{\infty\}$ defined by $H\left(z_{0}, z_{1}\right)=z_{0} / z_{1}$. The left action of $S^{1}$ on $S^{3}$ takes each Hopf fiber to itself, so preserves Hopf fibering, namely the fibers are the orbits of the left action of $S^{1}$ on $S^{3}$. If $G$ is a subgroup of $\operatorname{Isom}\left(S^{3}\right)$ which preserves the Hopf fibering then $S^{3} / G$ has an induced fibration from $S^{3}$. We call it the Hopf fibering of $S^{3} / G$. In this way, we may obtain Hopf fibrations on lens spaces. Basic details and background concerning elliptic structures and Hopf fibrations on elliptic manifolds may be found in [6] and [4] (section 3).

For $(z, w) \in S^{1} \times S^{1}$, we define $(z, w) \cdot(u, v)=(z u, w v)$ when $(u, v) \in$ $V_{1}$, and $(z, w) \cdot(u, v)=\left(z^{r} w^{p} u, z^{s} w^{q} v\right)$ when $(u, v) \in V_{2}$. This defines a torus action on $L$. Suppose $a$ and $b$ are relatively prime integers then $z \mapsto\left(z^{a}, z^{b}\right)$ is an embedding of $S^{1}$ into $S^{1} \times S^{1}$, and composing with the torus action defines an $S^{1}$-action on $L$. This action determines a Seifert fibering on $L$ in which $V_{1}$ and $V_{2}$ are union of fibers. On the solid torus $V_{1}$ the fibering has type $(a, b)$, and it has type $(r a+p b, s a+q b)$ on $V_{2}$. The associated Seifert fibration $\phi: L \rightarrow B=L / S^{1}$ has orbit space $B$ which is a 2-sphere with cone points of order $|a|$ and $|r a+p b|$. By making different choices of the type $(a, b)$ of the fibering on $V_{1}$, we may obtain infinitely many distinct Heegard fibering on $L$. Analyzing the orders $|a|$ and $|r a+p b|$ of the cone points on $B$ with the condition $r q-p s=-1$, one can easily deduce that $L(p, q)$ has a Heegaard fibering with no exceptional fibers if and only if $q= \pm 1(\bmod p)$.

In this paper, we give a certain uniqueness properties for the fiber of the Hopf fibration on lens spaces. 


\section{Hopf fibration}

From now on, we endow $L(p, q)$ with the Hopf fibering and assume that our sweepout of $L$ is selected so that each $P_{t}$ is a union of fibers.

LEMma 1. Let $L(p, q)$ be a lens space with $1 \leq q \leq p / 2$, which is Seifert-fibered with Hopf fibering. Let $P$ be a Heegaard torus which is a union of fibers, bounding solid tori $V$ and $W$. Suppose that a loop in $P$ is a longitude for $V$ and $W$. Then $q=1$. If $p>2$, then the loop is isotopic in $P$ to a fiber.

Proof. Let $\ell$ and $m$ be loops in $P$ which are respectively a longitude and a meridian of $V$, and so that $p \ell+q m$ is a meridian of $W$. If $c$ is any loop in $P$ which is a longitude for $V$, then (with one of its two orientations) $c$ has the form $\ell+k m$ in $H_{1}(P)$ for some $k$. The intersection number of $c$ with $p \ell+q m$ is $q-k p$. Since $1 \leq q \leq p / 2$, this can equal \pm 1 only if $(p, q, k)=(2,1,1)$ or $(p, 1,0)$, so $q=1$. When $p>2, k=0$ and so $c=\ell$. Since $q=1$, the Hopf fibering is nonsingular, so the fiber is a longitude for both $V$ and $W$. Since $p>2, c$ is the only longitude of $V$ that has intersection number \pm 1 with the meridian of $W$, so it must be isotopic in $P$ to a fiber.

TheOREM 2. Let $h: L(p, q) \rightarrow L(p, q)$ be a diffeomorphism isotopic to the identity with $h\left(P_{s}\right)=P_{t}$ where $0<s, t<1$. If $p>2$, then the image of a fiber of $P_{s}$ is isotopic in $P_{t}$ to a fiber.

Proof. Conjugating by a fiber-preserving diffeomorphism of $L(p, q)$ that moves $P_{s}$ to $P_{t}$, we may assume that $s=t$. Writing $P$ for $P_{t}$, let $V$ and $W$ be the solid tori that $P$ bounds. Let $\ell$ and $m$ be loops in $P$ as in the proof of lemma 1, and write $h_{*}: H_{1}(P) \rightarrow H_{1}(P)$ for the induced isomorphism.

Suppose first that $h(V)=V$. Since the meridian disk of $V$ is unique up to isotopy, we have $h_{*}(m)= \pm m$. Since $h$ is isotopic to the identity on $L(p, q)$ and $p>2, h$ is orientation preserving and induces the identity on $\pi_{1}(V)$. This implies that $h_{*}(m)=m$. Similar consideration for $W$ show that $h_{*}(p \ell+q m)=p \ell+q m$, so $h_{*}(\ell)=\ell$. Thus $h_{*}$ is the identity on $H_{1}(P)$ and the theorem follows for this case.

Suppose now that $h(V)=W$. Since $h$ is isotopic to the identity and reverses the sides of $P, h$ is orientation-reversing on $P$. Since $h$ must take a meridian of $V$ to one of $W, h_{*}(m)=\epsilon_{1}(p \ell+q m)$ where $\epsilon_{1}= \pm 1$. Writing $h_{*}(\ell)=r \ell+s m$, we find that $1=\ell \cdot m=-h_{*}(\ell) \cdot h_{*}(m)=$ $-\epsilon_{1}(q r-p s)$. The facts that $h$ is isotopic to the identity on $L, \ell$ generates $\pi_{1}(L)$, and $m$ is 0 in $\pi_{1}(V)$ imply that $r \equiv 1(\bmod p)$, so modulo $p$ we 
have $1 \equiv-\epsilon_{1} q$, forcing $q=1, \epsilon_{1}=-1$, and $h_{*}(m)=-p \ell-m$. Since $h$ carries a meridian of $W$ to one of $V$, we also have $h_{*}(p \ell+m)=\epsilon_{2} m$ where $\epsilon_{2}= \pm 1$. Subtracting, we find $h_{*}(p \ell)=p \ell+\left(1+\epsilon_{2}\right) m$. Since $p>2$ and $\epsilon_{2}= \pm 1$, we have $1+\epsilon_{2}=0$, so $h_{*}(\ell)=\ell$. Since $q=1, \ell$ has intersection number 1 with the meridian $p \ell+q m$ of $W$. Lemma 1 shows that $\ell$ is homotopic in $P$ to the fiber of the Hopf fibering. This proves the theorem.

\section{References}

[1] F. Bonahon, Diffeotopies des espaces lenticulaires, Topology 22 (1983), 305-314.

[2] J. Kalliongis and A. Miller, Geometric group actions on lens spaces, Kyungpook Math J. 42 (2002), 313-344.

[3] __ Actions on lens spaces which respect a Heegaard decomposition, Topology and its Applications 130 (2003) 19-55.

[4] D. McCullough and J. H. Rubinstein, The generalized Smale conjecture for 3mafolds with genus 2 one-sided Heegaard splittings, to appear in Trans. AMS.

[5] D. Rolfsen, Knots and links, Publish or Perish 1976.

[6] P. Scott, The geometries of 3-manifolds, Bull. London Math. Soc. 15 (1983), 401-487.

Department of Mathematics

Korea University

Seoul 136-701, Korea

E-mail: shong@semi.korea.ac.kr 